\title{
Foreword for the Special Section on Enabling Very High Penetration Renewable Energy Integration into Future Power Systems
}

\section{INTRODUCTION}

$\mathrm{R}$ enewable energy such as wind power, photovoltaic (PV) is regarded as one of the main solutions to achieve power system sustainability. In recent years, renewable energy has been experiencing fast development. In 2016, 24.5\% of the total power demand in the world was supplied by renewable energy, where 5.5\% was produced by wind power and PV [1]. It is estimated that by the end of 2017 the global wind power and PV capacity had reached about 565GW and $335 \mathrm{GW}$ respectively. Some power systems have already entered the era of high penetration of intermittent renewable energy integration. E.g., over $22.4 \%$ of the total power demand was supplied by intermittent renewables in Spain in 2015. It is reported that on July 11, 2015, the wind power generation reached $140 \%$ of the whole country's demand in Denmark [2]. In several provinces of China such as Gansu, Qinghai, and Xinjiang, the installed capacity of intermittent renewable energy have already occupied over $30 \%$ of the generation capacity [3].

Countries around the world have set aggressive goals for high penetration of renewable energy in future power systems. The integration of high penetration of renewable energy will fundamentally change the configuration of power system [4]. The way that power system balances the generation and load will change in both planning in long-time scale and operation in short-time scale, and in both transmission and distribution networks. The intermittency of renewables will raise power system security concern, ancillary services adequacy concern and economic issues. Hence, high penetration of renewable energy integration will bring significant challenges to future power systems. Failing to address these challenges may result in large amounts of wind power and PV curtailment and jeopardize the economics of renewable energy.

Different countries have funded more and more projects for high penetration renewable energy integration research through certain departments, such as National Science Foundation (NSF) in the US, the biggest research and innovation programme $\mathrm{H} 2020$ in European Union, and National Science Foundation of China (NSFC). Take China as an example, in 2016 the National Key Research and Development Program of China approved a project entitled Fundamental Theory of Planning and Operation for Power Systems with High Share of Renewable Energy Generations. The overall objective of this project is to study the basic theory and method of planning and operation of high penetration renewable energy power systems and to provide standardized systems for a high share of renewable energy integration.

This Special Section is dedicated to providing innovative insights on future power systems accommodating high penetration of renewable energy. We follow a two-tier review process. First, about 300 two-page extended abstracts were received and reviewed by the guest editorial board. Around 100 papers were invited for eight-page full paper submission. Finally, we accept 24 papers in this special section. Since the challenges brought by high penetration of renewable energy are multifaceted, we group these papers into seven schemes: 1) stochastic dependencies modeling; 2) power system forecasting; 3) power system flexibility; 4) transmission system related issues; 5) distribution system \& microgrid operation; 6) power system frequency control; 7) power system oscillation analysis. A brief summary of these paper is presented in the following.

\section{ABOUT THE PAPERS}

\section{A. Stochastic Dependencies Modeling}

The power system with high penetration of renewable energy integration presents great uncertainties. The dependencies among these uncertainties from the load and renewable energy have great impact on power system operation, stability, and power quality. Thus, accurate modelling of these dependencies is very important and influential in the high renewable energy penetrated situations.

K. N. Hasan and R. Preece investigated the influence of stochastic dependence on the small-disturbance stability of the power system with high penetration of renewable energy [5]. Global sensitivity analysis (GSA) was applied to identify and rank the critical uncertainties that most affect the damping of the most critical oscillatory mode. Results showed that Gaussian copula is the most suitable approach and has consistently low error even at higher levels of renewable energy penetration into the power system. X. Xu et al. performed a priority ranking of renewable energy variabilities that will affect the voltage stability of power systems also based on GSA [6]. The stochastic response surface method (SRMS) improved the computation efficiency of the GSA based evaluation method. S. Pukhrem et al. proposed a MonteCarlo simulation based probabilistic power quality (PQ) assessment method of the low voltage distribution network with high PV integration [7]. The PQ impact metrics included small variations and abnormal event and were assessed in terms of site and system PQ indices. Case studies showed that increase of PV integration results in high overvoltage risk and the PV integration can reduce the voltage unbalance as compared with no or low PV penetration.

\section{B. Power System Forecasting}

Centralized and distributed integration of renewable energy will aggravate the variabilities and nonlinear correlations of wind/solar resources in multiple scales, and thus pose challenges to the forecasting of loads and renewable power generation. 
Y. Wang et al. developed a data-driven probabilistic net load forecasting method specifically for a high penetration of distributed PV generation [8]. Correlation analysis based on copula theory is conducted on the distributions and dependencies of the forecasting errors to generate a probabilistic net load forecast. Y. Chen et al. proposed a datadriven approach for scenario generation using generative adversarial networks to capture renewable energy production patterns in both temporal and spatial dimensions for a large number of correlated resources [9]. J. Yan et al. presented a multi-scale and reginal wind power forecasting method to capture the variable correlation patterns of wind and its power output by establishing a multi-to-multi mapping network and the use of ensemble stacked denoising auto-encoder [10].

\section{Power System Flexibility}

Lack of flexibility will result in renewable energy curtailment. One paper discussed the flexibility evaluation method; while two papers exploited the flexibility of power system for the perspective of the usage of energy storage and the technology of dynamic line rating.

Z. Lu et al. discussed the problem of power system planning with consideration of renewable energy curtailment [11]. A probabilistic flexibility evaluation method was proposed which can reflect the direction, amount, frequency and consequence of lack of flexibility. Thanks to the linear relationship of the indices with the renewable energy curtailment, the evaluation method can be conveniently applied in power system planning with very high penetration of renewables. X. Dui et al proposed a two-stage method to determine the optimal power and capacity of battery energy system [12]. The results showed that the coordination between battery energy system and wind farms can significantly reduce the operation cost and renewable energy curtailment. F. Teng et al analyzed the benefits of dynamic line rating in the transmission system [13]. A scheduling model with consideration of multiple sources of uncertainty including wind generation, line ratings, and line outrages was proposed. The case studies demonstrated the benefits of dynamic line rating in supporting cost effective integration of high penetration of wind generation.

\section{Transmission System Related Issues}

Great uncertainty and variability of renewable energy and the change of gird configuration challenge the traditional ways of operation and planning, such as unit commitment, optimal dispatch, and reliability analysis.

J. Shi and S. S. Oren proposed a stochastic unit commitment model considering the topology control recourse for transmission switching [14]. It was proven that transmission switching can not only reduce the objective value of direct current optimal power flow (DCOPF) when congestion exists but also reduce unit commitment cost when congestion does not exist. M. Zhou et al developed a distributed dispatch approach for bulk AC/DC hybrid systems, where the day-ahead unit commit problem was decomposed into a master problem to determine the day-ahead transmission plan for high voltage direct current (HVDC) tie-line and various parallel sub-problems for regional dispatch [15]. D. Apostolopoulou et al examined the optimal dispatch for a cascade hydroelectric power system with consideration of the uncertainty of net load demand to maximize the head levels of each dam, and minimize the spillage effects [16]. The power system reliability was addressed M. Fan $e t$ al, where a novel generation rescheduling algorithm was proposed to mitigate the variations of branch power flows and relieve the overload probability [17]. The strong volatility of load and intermittent renewable generation may also lead to the tension of operating reserve. Y. Lin et al. proposed a multi-state model to quantitatively analyze the upward and downward reserve capacity of wind farms [18].

\section{E. Distribution System \& Microgrid Operation}

Distributed renewable energy integration especially for PV generation has a great impact on the power quality and stability of distribution system and microgirds. Several papers discussed low-voltage network related issues from various aspects including electricity market, voltage control, stability analysis, etc.

X. Yan et al. proposed a novel Locational Marginal Pricing (LMP) model to incentive energy storage (ES) to reduce system congestion cost, where the pricing was derived by evaluating ES impact on the network power flows and congestions from the loss and congestion components in LMP [19]. This work can further increase network flexibility and the capability of networks to accommodate increasing PV penetration. L. Wang et al. proposed a real-time PV inverters and battery energy storage system (BESS) coordinated control framework for voltage regulation of a weak distribution network with large-scale PV integration [20]. The control strategy can help both utilities and PV owners for PV integration. Y. Chai et al. provided a double-layer voltage control strategy based on the distribution network partition [21]. It combined the cluster autonomous optimization and distributed inter-cluster coordination optimization in different time scales. D. Choi et al. proposed a virtual multi-slack (VMS) droop control for the stand-alone microgrid [22]. In the proposed control framework, one physical slack generator directly controls the magnitude and phase angle of its bus voltage and the other generators indirectly control the magnitudes and phase angles of their bus voltages by using the VMS droop control. It results in the proper load sharing between the generators. Y. Li et al. presented a formal analysis for the stability assessment of the networked microgrids with high penetration of distributed energy resources (DERs) [23]. The analysis with mathematical rigor directly computes the bounds of all possible dynamic trajectories and provides stability information unattainable by traditional time-domain simulations or direct methods. Results showed that the analysis can efficiently evaluate the impacts of disturbances on networked microgrid dynamics and how far the networked microgrid system is from its stability margins.

\section{F. Power System Frequency Control}

The performance of frequency response is one of the most important indexes in power system operation. However, under the very high penetration of renewable energy, the power system may suffer from low inertia and declining frequency. To solve this problem, two papers proposed ideas both from theoretical derivation and industry perspective. 
D. Yang et al proposed a temporary frequency support scheme of DFIG that can improve the frequency nadir while ensuring rapid frequency stabilization. According to the analysis and test results, the proposed scheme performed well, particularly for high wind power penetration levels [24]. Y. Liu et al assessed the frequency response of U.S. power grid with an industry-provided full-detail interconnection model [25]. The paper also discussed the practical solutions to address the declining frequency response in the U.S.

\section{G. Power System Oscillation Analysis}

Power electronic converters and AC/DC grid have been increasing with more and more renewable energy being integrated into power systems. The dynamic interaction between the AC/DC grid and power electronic converters make the oscillatory stability analysis much more complex.

J. Ying et al studied the impact of inertia control of DFIGbased wind turbine on electromechanical oscillation damping via a linearized model of wind turbine [26]. Through the analysis, the cause and effect of oscillation damping were explained from a general perspective. C. Liu et al. proposed a line modal potential energy (LMPE) method for the inter-area oscillation analysis and damping control of doubly fed induction generators (DFIGs) from the network perspective [27]. The proposed method can be used for sub-mode analysis and oscillation suppression. $\mathrm{H}$. Liu et al. presented the concept of unified $d q$-frame impedance network model (INM), with which, different converters, as well as traditional generators/HVDCs, can be incorporated to form an integrated $s$-domain model of a practical system [28]. Then, a new criterion is then proposed to quantify the oscillatory stability by analyzing the frequency characteristics of the determinant of the matrix.

\section{ACKNOWLEDGEMENTS}

We would like to appreciate all authors for their innovative and in-depth works. We are grateful to all reviewers for their thoughtful comments improving the quality of the papers. We also wish to express our gratitude for Prof. Nikos Hatziargyriou's support and suggestions during the whole process.

\section{Chongqing Kang \\ Tsinghua University, China \\ cqkang@tsinghua.edu.cn}

$$
\begin{aligned}
& \text { Zhe Chen } \\
& \text { Aalborg University, Denmark } \\
& \text { zch@et.aau.dk }
\end{aligned}
$$

\author{
Ning Zhang \\ Tsinghua University, China \\ ningzhang@tsinghua.edu.cn \\ Oriol Gomis-Bellmunt \\ Polytechnic University of Catalonia, Spain \\ ogomis@irec.cat
}

Mike Barnes
Manchester University, UK mike.barnes@manchester.ac.uk

Jie Yan

North China Electric Power University, China

yanjie@ncepu.edu.cn

Weihao Hu

Aalborg University, Denmark

whu@et.aau.dk

Kai Sun

University of Tennessee, USA

kaisun@utk.edu

\section{REFERENCES}

[1] Renewable Energy Policy Network for the 21st Century, "Renewables 2017 global status report”, [Online]. Available:. $\quad$ http://www.ren21.net/wp-content/uploads/2017/06/178399_GSR_2017_Full_Report_0621_Opt.pdf.

[2] The Guardian, "Wind power generates $140 \%$ of Denmark's electricity demand” [Online]. Available:. https://www.theguardian.com/environment/2015/jul/10/denmark-windwindfarm-power-exceed-electricity-demand.

[3] China Energy Research Society, "China's energy development report 2016,” Beijing, China,2016.( in Chinese)

[4] B. Kroposki et al., "Achieving a 100\% Renewable Grid: Operating Electric Power Systems with Extremely High Levels of Variable Renewable Energy," IEEE Power and Energy Magazine, vol. 15, no. 2, pp. 61-73, March-April 2017.

[5] K N. Hasam and R. Preece, "Influence of Stochastic Dependence on Small-Disturbance Stability and Ranking Uncertainties," IEEE Trans. Power Systems, vol. 33, no. 3, pp. ??-??, May, 2018(to be confirmed).

[6] X. Xu, Z. Yan, M. Shahidehpour, H. Wang, and S. Chen, "Power System Voltage Stability Evaluation Considering Renewable Energy with Correlated Variabilities," IEEE Trans. Power Systems, vol. 33, no. 3, pp. ??-??, May, 2018.

[7] S. Pukhrem, M. Basu, and M. Conlon, "Probabilistic Risk Assessment of Power Quality Variations and Events under Temporal and Spatial Characteristic of Increased PV Integration in Low Voltage Distribution Networks," IEEE Trans. Power Systems, vol. 33, no. 3, pp. ??-??, May, 2018.

[8] Y. Wang, N. Zhang, Q. Chen, D. Kirschen, P. Li, and Q. Xia, "DataDriven Probabilistic Net Load Forecasting with High Penetration of Behind-the-Meter PV," IEEE Trans. Power Systems, vol. 33, no. 3, pp. ??-??, May, 2018.

[9] Y. Chen, Y. Wang, D. Kirschen, and B. Zhang, "Model-Free Renewable Scenario Generation Using Generative Adversarial Networks," IEEE Trans. Power Systems, vol. 33, no. 3, pp. ??-??, May, 2018.

[10] J. Yan, H. Zhang, Y. Liu, S. Han, L. Li, and Z. Lu "Forecasting High Penetration of Wind Power on Multi-scales Using Corrected Multiple NWPs," IEEE Trans. Power Systems, vol. 33, no. 3, pp. ??-??, May, 2018.

[11] Z. Lu, H. Li, and Y. Qiao, "Probabilistic Flexibility Evaluation for Power System Planning Considering Its Association with Renewable Power Curtailment," IEEE Trans. Power Systems, vol. 33, no. 3, pp. ????, May, 2018.

[12] X. Dui, G. Zhu, L. Yao, " Two-stage optimization of battery energy storage capacity to decrease wind power curtailment in grid-connected wind farms," IEEE Trans. Power Systems, vol. 33, no. 3, pp. ??-??, May, 2018.

[13] F. Teng, R. Dupin, A. Michiorri, G. Kariniotakis, Y. Chen, and G. Strbac, "Understanding the Benefits of Dynamic Line Rating under Multiple Sources of Uncertainty," IEEE Trans. Power Systems, vol. 33, no. 3, pp. ??-??, May, 2018.

[14] J. Shi and S. Oren, "Stochastic Unit Commitment with Topology Control Recourse for Power Systems with Large-Scale Renewable Integration," IEEE Trans. Power Systems, vol. 33, no. 3, pp. ??-??, May, 2018. 
[15] M. Zhou, J. Zhai, G. Li, and J. Ren "Distributed Dispatch Approach to the Bulk AC/DC Hybrid System with High Wind Power Penetration," IEEE Trans. Power Systems, vol. 33, no. 3, pp. ??-??, May, 2018.

[16] D. Apostolopoulou, Z. D. Gr`eve, and M. McCulloch "Robust Optimisation for Hydroelectric System Operation under Uncertainty," IEEE Trans. Power Systems, vol. 33, no. 3, pp. ??-??, May, 2018.

[17] M. Fan, K. Sun, D. Lane, W. Gu, Z. Li, and F. Zhang, "A Novel Generation Rescheduling Algorithm to Improve Power System Reliability with High Renewable Energy Penetration," IEEE Trans. Power Systems, vol. 33, no. 3, pp. ??-??, May, 2018.

[18] Y. Liu, Y. Ding, Y. Song, and C. Guo, "A Multi-State Model for Exploiting the Reserve Capability of Wind Power," IEEE Trans. Power Systems, vol. 33, no. 3, pp. ??-??, May, 2018.

[19] X. Yan, C. Gu, F. Li, and Z. Wang, "LMP-based Network Pricing for Energy Storage in Local Market to Facilitate PV Penetration," IEEE Trans. Power Systems, vol. 33, no. 3, pp. ??-??, May, 2018.

[20] L. Wang, F. Bai, R. Yan, and T. K. Saha, "Real-Time Coordinated Voltage Control of PV Inverters and Energy Storage for Weak Networks with High PV Penetration," IEEE Trans. Power Systems, vol. 33, no. 3, pp. ??-??, May, 2018.

[21] Y. Chai, C. Wang, L. Guo, and Z. Zhao, "Network Partition and Voltage Coordination Control for Distribution Networks with High Penetration of Distributed PV Units," IEEE Trans. Power Systems, vol. 33, no. 3, pp. ??-??, May, 2018.

[22] D. Choi, J. Park, and S. H. Lee, "Virtual Multi-Slack Droop Control of Stand-Alone Microgrid with High Renewable Penetration Based on Power Sensitivity Analysis," IEEE Trans. Power Systems, vol. 33, no. 3, pp. ??-??, May, 2018.

[23] Y. Li, P. Zhang, and P. B. Luh, "Formal Analysis of Networked Microgrids Dynamics," IEEE Trans. Power Systems, vol. 33, no. 3, pp. ??-??, May, 2018.

[24] D. Yang, J. Kim, Y. C. Kang, E. Muljadi, N. Zhang, J. Hong, S. Song, and T. Zheng, "Temporary Frequency Support of a DFIG for High Wind Power Penetration," IEEE Trans. Power Systems, vol. 33, no. 3, pp. ????, May, 2018.

[25] Y. Liu, S. You, J. Tan, Y. Zhang, and Y. Liu, "Frequency Response Assessment and Enhancement of the U.S. Power Grids towards ExtraHigh Photovoltaic Generation Penetrations-an Industry Perspective," IEEE Trans. Power Systems, vol. 33, no. 3, pp. ??-??, May, 2018.

[26] J. Ying, X. Yuan, J. Hu, and W. He, "Impact of Inertia Control of DFIGbased WT on Electromechanical Oscillation Damping of SG," IEEE Trans. Power Systems, vol. 33, no. 3, pp. ??-??, May, 2018.

[27] C. Liu, G. Cai, D. Yang, C. Liu, and Z. Sun, "Oscillations Analysis and Wide-area Damping Control of DFIG for Renewable Power System using Modal Potential Energy," IEEE Trans. Power Systems, vol. 33, no. 3, pp. ??-??, May, 2018.

[28] H. Liu, X. Xie, and W. Liu, "An Oscillatory Stability Criterion based on the Unified $d q$-Frame Impedance Network Model for Power Systems with High-Penetration Renewables," IEEE Trans. Power Systems, vol. 33, no. 3, pp. ??-??, May, 2018. 